\title{
La vinculación entre modelo de desarrollo y estrategia de inserción en la Argentina democrática (1983-2011) *
}

Esteban Actis **

María Elena Lorenzini ***

Julieta Zelicovich $* * * *$

\section{Resumen}

La relación entre el modelo de desarrollo y la estrategia de inserción internacional constituye un vínculo clave para la comprensión de la política exterior. Sin embargo, la misma ha sido poco considerada en los aná-

\footnotetext{
* Este trabajo se inscribe en el marco del Proyecto: "Modelos de desarrollo e inserción internacional. Aportes para el análisis de la política exterior argentina desde la redemocratización (1983-2011)”, financiado por la Agencia Nacional de Investigaciones Científicas y Técnicas de la República Argentina (PICT-2011-0681) Res. 140/12, dirigido por Anabella Busso. El trabajo es en coautoría y el orden en el que se mencionan los autores es estrictamente alfabético.

** Becario Posdoctoral del Consejo Nacional de Investigaciones Científicas y Técnicas (CONICET).

*** Investigadora del Consejo Nacional de Investigaciones Científicas y Técnicas (CONICET).

**** Investigadora del Consejo Nacional de Investigaciones Científicas y Técnicas (CONICET).
}

Código de referato: SP.220.XLI/17

http://dx.doi.org/10.22529/sp.2017.41.04

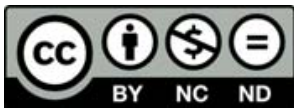

STUDIA POLITICAE \$s Número 41 otoño 2017

Publicada por la Facultad de Ciencia Política y Relaciones Internacionales, de la Universidad Católica de Córdoba, Córdoba, República Argentina. 
lisis de las relaciones internacionales contemporáneas. En este artículo nos proponemos analizar la definición de ambos conceptos, buscando identificar los canales a través de los cuales se articulan e impactan en la política externa. El artículo realiza un análisis bibliográfico y documental, donde la discusión teórica-conceptual que vincula modelo de desarrollo-estrategia de inserción se acompaña del análisis de datos empíricos correspondientes a la política exterior argentina en el período 1983-2011.

Palabras claves: Modelo de Desarrollo - Estrategia de Inserción Internacional - Política Exterior - Argentina

\begin{abstract}
The relationship between the development strategy and the definition of the way by which a country defines its participation in the international relations is a key link for understanding the foreign policy. However, this linkage has been understudied in the contemporary international relations. In this article we analyze these key concepts definitions, identifying their bond and the way they impact on foreign policy. We apply bibliographic and documental analysis, and link the theoretical discussion with the analysis of empirical data on Argentine foreign policy during the period 1983-2011.
\end{abstract}

Key words: Development strategy - International Strategy - Foreign Policy - Argentina

\title{
Introducción
}

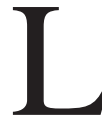

A articulación entre el modelo de desarrollo y la estrategia de inserción internacional es una de las vinculaciones menos exploradas en la literatura especializada en relaciones internacionales, pero no por ello menos importante. En este sentido, es nuestro objetivo aportar instrumentos para clarificar este vínculo desde el análisis del modelo de desarrollo.

Esta relación, poco estudiada dentro de los análisis de política exterior, ${ }^{1}$ resulta a nuestro criterio fundamental para la comprensión de las variaciones y continuidades del comportamiento externo de un Estado.

1 Algunos de quienes han trabajo esta relación son RAPOPORT (2006), RAPOPORT y SPIGUEL (2003), Míguez (2013), RAPOPORT y Míguez (2015). Estos autores han trabajado la articulación entre los modelos de acumulación y la política exterior desde una mirada preeminentemente histórica y no internacionalista. 
Hasta 1983, la erraticidad del accionar externo de la Argentina se explicaba a partir de la variable régimen político ${ }^{2}$ en relación a la alternancia entre gobiernos autoritarios y gobiernos democráticos. Esta hipótesis haría suponer que una vez consolidada la democracia, la Argentina exhibiría mayores continuidades en su política exterior (PE). Sin embargo, en la Argentina redemocratizada la oscilación de la PE continuó estando presente. En ese sentido, sostenemos que las variaciones y rupturas de los modelos desarrollo y su vinculación con la estrategia de inserción internacional de uno de los factores claves para explicar la discontinuidad de la PE.

El artículo se presenta como una investigación cualitativa basada en el análisis bibliográfico y documental. El recorte temporal obedece a la aspiración de comprender las variaciones y continuidades de la política exterior argentina desde el retorno de la democracia, signado por el comienzo del gobierno de Raúl Alfonsín, hasta la finalización del primer mandato del gobierno de Cristina Fernández de Kirchner. ${ }^{3}$ A tales fines se ha realizado una profunda revisión de la literatura sobre política exterior argentina en el período 1983-2011, tanto desde la perspectiva internacionalista como economicista. La discusión teórica-conceptual se acompaña del análisis de datos empíricos correspondientes al período histórico seleccionado. El trabajo contribuye al debate acerca de cómo estos conceptos - modelo de desarrollo y estrategia de inserción internacionalson abordados en los estudios, enfatizando las complejidades de su operacionalización. Se trata de brindar herramientas para tender puentes entre la teoría y la empiria, siendo esta la contribución esperada del artículo a los estudios disciplinares. Por lo tanto, el trabajo no pretende brindar un compendio acabado de las PE del período 1983-2011 sino indicios acerca de cómo estas dos variables han repercutido transversalmente en el accionar externo de la Argentina.

En el primer apartado se discute la definición de modelo de desarrollo a partir de tres dimensiones analíticas: productiva, financiera y social. Una

2 Por un lado, un grupo de trabajos sostuvo que las transiciones a la democracia generaban modificaciones sobre la política externa (HuRRell, 1995; HuRrel, 2003; MoraVcSIK, 2002; Doyle, 1983; Doyle, 1986; Cervo, 2003; RAPOPORT y SPIguel, 2003), entre los más destacados. Por el otro, se planteaba que no existía una relación de causalidad directa entre cambio de régimen y dicha política pública, aplicable a todos los casos de transiciones democráticas (Sombra SARAIVA, 2003; BenoIT, 1995/6; GAUBATZ, 1996).

3 En función de priorizar el análisis sobre períodos presidenciales completos, y debido a que la elaboración de este trabajo se sustanció durante el año 2015, es que solo se incluyó el primer gobierno de Fernández de Kirchner. 
vez identificadas, se considera, además, su articulación con la definición de estrategia de inserción internacional. En el segundo apartado, siguiendo dichas dimensiones, se analizan tres tipos ideales de modelos de desarrollo, a saber: el estructuralista, el neoliberal y el neodesarrollista. Por último, se presenta la aplicación de estos tipos y su vinculación con la estrategia de inserción al caso Argentino durante tres períodos: Alfonsín (1983-1989), Menem (1989-1999), Kirchner (2003-2007) y Fernández de Kirchner (2008-2011). ${ }^{4}$

\section{Modelo de desarrollo: aproximación teórica en tres dimensiones y su articulación con la estrategia de inserción internacional}

La idea de 'desarrollo' ha sido un eje estructurante en la conformación del pensamiento latinoamericano. Tuvo un crecimiento importante con la corriente cepalina ${ }^{5}$, fue resignificada durante los debates en el período de la hegemonía neoliberal y tuvo un nuevo momento de auge hacia principios de la primera década del siglo XXI con la proliferación de estudios sobre el neodesarrollismo. Su alcance trasciende las definiciones económicas y se convierte, entre otros, en un estructurante importante de la estrategia de inserción internacional y de la PE del país.

El modelo de desarrollo ${ }^{6}$ refiere a la manera en la que se articulan la política y la economía entre el Estado y el mercado en un contexto histórico determinado. A cada modelo le corresponde un modo de producción, de distribución de la riqueza y una estrategia de inserción internacional. Resultado de ello serán los diferentes patrones de tipo de cambio, las regulaciones del comercio exterior, las demandas en las negociaciones exteriores y la posición que el actor asuma frente a otros temas como la educación, los recursos tecnológicos y del conocimiento, las instituciones, etc. (Ferrer, 2011; Ffrench Davis, 2001; Kaldor y Mirrlees, 1962; CEPAL, 1998; Biel-

${ }^{4}$ La continuidad existente entre los gobiernos de Kirchner y Fernández de Kirchner justifica su clasificación como un único período.

5 Se entiende por “corriente cepalina” los trabajos desarrollos entre los años cincuenta y ochenta del siglo XX al interior de la Comisión Económica para América Latina (CEPAL), cuyos principales referentes intelectuales fueron Raúl Prebish, Celso Furtado, Osvaldo Sunkel, entre otros. Para más información véase Di FILIPPO (2007), BÁRCENA y PRADO (2015).

6 Si bien autores como Devlin y Moguillansky (2009) distinguen modelo y estrategia de desarrollo, no es objeto de este trabajo introducirse en dicho debate, siendo que tomaremos ambos conceptos como equivalentes. 
chowsky, 2009; Bresser Pereira, 2007; Di Pace, 1992). El modelo de desarrollo es así "una construcción social, que supone la participación del conjunto de los actores en la definición de los componentes” (Ferrer, 2005). Los contextos en los cuales se dan estas decisiones resultan fundamentales en tanto determinan la permisibilidad y viabilidad de cada acción.

Desde la perspectiva que orienta este trabajo, se identifican tres dimensiones analíticas, donde cada una de las cuales reúne un conjunto de variables que resultan de utilidad para la clasificación de los modelos de desarrollo y su vinculación con las estrategias de inserción internacional.

En primer lugar, se señalan las definiciones del sistema productivo doméstico y su articulación con la inserción productiva internacional. Esto comprende, en primer término, si se prioriza un esquema productivo basado en las ventajas comparativas — dadas - o si en cambio el principal énfasis de las políticas públicas recae en la construcción de ventajas competitivas - creadas - . La segunda cuestión, alude al modo a través del cual la estructura productiva de un país relaciona la oferta de sus bienes y servicios que vinculan comercialmente el mercado interno y el internacional (Ortiz Cruz, 2010; Frenkel, 2003). Se define en este eje el grado de intervención pública sobre los niveles de apertura externa, teniendo en cuenta la relación entre la demanda doméstica e internacional y la estructura productiva; también, los instrumentos de política comercial externa aplicados y los criterios de inversión externa directa (IED).

En segundo lugar, se encuentra el manejo de las relaciones entre el Gobierno, el sector financiero y el aparato económico. Este núcleo apunta a las condiciones de estabilidad macroeconómica, a las modalidades de intervención y manejo de las políticas monetarias y fiscales (Bresser Pereira, 2007; Ffrench Davis, 2001). En estas cuestiones entra en tensión el binomio crecimiento-estabilidad monetaria, ya que niveles de crecimiento acelerados tienden a generar presiones inflacionarias y, viceversa, la implementación de políticas de control de precios, pueden ralentizar el crecimiento de una economía. Por este motivo, referentes centrales de estas variables son el tipo de cambio, la tasa de interés, la tasa de inflación o índice de precios, el ahorro interno y la relación entre financiamiento interno y financiamiento externo.

En tercer lugar, se mencionan las condiciones a partir de las cuales el modelo económico se vincula con la dimensión social. Esto alude al rol que se le asigna al Estado respecto del mercado para llevar adelante la distribución del ingreso, las políticas sociales de reducción de la pobreza, creación de empleo, educación y salud (Bresser Pereira, 2007; Ortiz Cruz, 2010; Ffrench Davis, 2001); también las políticas del Estado en la genera- 
ción de externalidades positivas vinculadas a las definiciones productivas, económicas, monetarias y financieras, tales como la protección de la propiedad intelectual, la promoción de la investigación y desarrollo, y la infraestructura vinculada a tales procesos - energía, transporte, comunicaciones, etc.- En otros términos, se observan los niveles de gasto público, sus prioridades y su implementación.

Por otra parte, entendemos que la estrategia de inserción internacional expresa la elección del esquema central de un conjunto de orientaciones y lineamientos de la PE que un Estado decide poner en práctica para vincularse con sus pares en el sistema internacional tanto en la dimensión política y de seguridad como económica (Lorenzini, 2011, p. 43). En tanto estrategia, supone una serie de acciones planificadas que se desarrollan de cara a un objetivo. Es una suerte de brújula que permite interpretar el rumbo de la $\mathrm{PE}$. Algunos de los ejes que diferencian las estrategias de inserción internacional refieren a la manera en la que se percibe al orden internacional como oportunidad o como amenaza, lo que conduce a un posicionamiento de adhesión, reforma o impugnación. El modo en el que se valoran las asimetrías y la interdependencia en las relaciones externas hace que se privilegien las relaciones con las potencias hegemónicas o que se prefieran los vínculos de asociación y acercamiento en el eje Sur-Sur.

En los párrafos anteriores se puede apreciar que el modelo de desarrollo, al igual que la estrategia de inserción, implica un esquema de orientaciones y lineamientos en su relación con el sistema internacional. Estos remiten a la compatibilidad o incompatibilidad de la política económica doméstica — comercial, financiera y social- con la política económica externa. La relación entre ambos esquemas de orientaciones y lineamientos - los del modelo de desarrollo y los de la estrategia de inserción internacional- es la que permite comprender de qué manera interactúan, afectando la PE.

Para ello es preciso considerar, dentro de la primera dimensión del modelo de desarrollo, los imaginarios, cosmovisiones y orientaciones comprendidos en la definición y el rol asignado a la apertura externa — comercial y de inversiones- - Dentro de la segunda dimensión, tal articulación podrá observarse, en cambio, en las apreciaciones del modelo respecto del ahorro externo y el modo de vinculación con el sistema financiero internacional. Por último, la vinculación entre ambos conceptos en la tercera dimensión vendrá contenida dentro de las consideraciones que el modelo realice respecto de la generación o no de externalidades positivas para el desarrollo a partir de las relaciones con otros Estados — por ejemplo, integración, cooperación internacional, etc.-. 


\section{Tipos ideales de modelos de desarrollo}

Siguiendo a Devlin y Moguillansky sostenemos que "la principal línea divisoria en relación con el carácter de las estrategias de desarrollo es el grado de intervención pública orientada a lograr las metas establecidas, así como la importancia que se otorga a la aceleración del proceso de transformación productiva, más allá del ritmo natural del mercado” (2009, p. 100). Acorde a ello, en un polo se ubicarían aquellos que sostienen "que el libre juego del mercado resolverá los problemas económicos y que una política macroeconómica 'sana' genera incentivos suficientes como para que los empresarios lideren por sí mismos una transformación productiva basada en las ventajas comparativas internacionales del país" (Devlin y Moguillansky, 2009, p. 100-101). En el polo opuesto, se encontrarían aquellos otros "que desconfían de algunas de las señales del mercado y de que las ventajas comparativas estáticas por sí solas puedan impulsar la transformación productiva a un ritmo adecuado para lograr la convergencia con los países desarrollados" y que afirman entonces que son necesarias intervenciones por parte del gobierno para alcanzar tal transformación productiva (Devlin y Moguillansky, 2009, p. 100-101).

En las ideas y teorías económicas, el primer tipo de estrategia forma parte del paradigma ortodoxo también llamado 'paradigma monetarista' o 'neoliberal', mientras que la segunda es propugnada por el 'paradigma heterodoxo' o 'estructuralista' latinoamericano — políticas desarrollistas - o bien su revisión actual, conocida como 'neoestructuralismo' —políticas neodesarrollistas_-, ${ }^{7}$ cuyos teóricos principales forman parte de la denominada corriente 'cepalina'.

\section{2.a. Dimensión productiva}

En la primera de las dimensiones, la de la estructura productiva y la inserción productiva internacional, los modelos ortodoxos postulan al mercado

\footnotetext{
7 Sin embargo, el neodesarrollismo según BRESSER-PEREIRA (2007) no debería encasillarse de forma pura en ninguno de los grupos porque puede ser calificado como una estrategia impura o como 'tercer discurso' ubicado entre el discurso del nacional desarrollismo y el de la ortodoxia convencional, dado que el Estado y el mercado son instituciones de la sociedad; son sus instrumentos de acción colectiva y son las herramientas principales de cada sociedad para alcanzar sus objetivos. El instrumento fundamental es el Estado; el mercado lo complementa. Por su parte, entre neodesarrollistas y neoestruturalistas debe reconocerse un énfasis diferente en los sectores productivos que priorizan en sus estrategias de desarrollo.
} 
como el principal actor promotor de la dinámica económica y le asignan al Estado una participación más bien, escasa, especialmente como garante de las reglas de juego. Una macroeconomía sana es una condición suficiente para lograr los objetivos productivos. Se privilegian las ventajas comparativas y la apertura comercial. Respecto de la IED, la misma es considerada deseable, puesto que se asume un efecto derrame inmediato hacia el resto de la sociedad y el sistema productivo en su conjunto. En función de ello se estimulan contextos flexibles para la recepción/atracción de la IED. En particular se prioriza generar una llamada 'seguridad jurídica' como mecanismo de atracción, complementándose en segundo lugar, con otros incentivos económicos (Oman, 2000).

Los modelos heterodoxos tradicionales, en cambio, piensan que el rol clave en la promoción del desarrollo económico le corresponde al Estado - concebido como la herramienta principal de la acción colectiva- a través de la estrategia de sustitución de importaciones (ISI) y con el objetivo de establecer las bases industriales del país (Bielchowsky, 2009; Bresser Pereira, 2007; Ortiz Cruz, 2010). En esa dirección y basándose en la teoría keynesiana, la estabilidad macroeconómica no constituye una prioridad en la agenda desarrollista puesto que se tolera cierto nivel de déficit fiscal como una de las vías - privilegiada junto con el ahorro forzoso por parte del Estado frente a las inversiones externas - para financiar la industrialización de las empresas que integran la estructura productiva del país. En consonancia con la estrategia ISI, promueven la generación de ventajas competitivas y, en función de ello, asumen una actitud proteccionista a la que se suma un pesimismo exportador que rechaza la apertura de la economía del país frente al mercado externo (Bresser Pereira, 2007).

Los modelos neodesarrollistas comparten con el desarrollismo tradicional la idea de que el Estado debe jugar un rol estratégico en materia de inversión y política industrial, pero sin caer en la dicotomía: Estado o mercado. En un contexto internacional de características diferentes a los años 50 y 60 , los neodesarrollistas le asignan al sector externo un papel importante en la búsqueda del desarrollo, lo que se hace visible en el aliento a las exportaciones con valor agregado y en un control moderado de la cuenta de capitales (Bresser Pereira, 2007). Destacan la importancia de la alianza público-privado, en el sentido de adoptar medidas que estimulen mejor al sector privado a tomar decisiones compatibles con las metas de la estrategia en cuestión. El núcleo de dicha alianza no son las decisiones concretas que la sustentan, sino las percepciones compartidas por el sector privado y el sector público sobre el rumbo económico y productivo que debe adoptarse. Como argumenta Stiglitz (1998) las estrategias de desarrollo con un enfoque proactivo de transformación surgen, en primer lugar, a partir de un 
diagnóstico y evaluación, tanto desde el gobierno como de los sectores empresariales, de las oportunidades clave para mejorar el posicionamiento del país en el mercado mundial, con una proyección a mediano y largo plazo e identificando las restricciones primarias que es preciso superar, reducir o eliminar a fin de aprovechar dichas oportunidades.

El neodesarrollismo - también conocido como neoestructuralismo- puede caracterizarse entonces como un conjunto de políticas públicas que apuntan a eliminar o compensar, a través de la intervención del Estado, las fallas del mercado, ejerciendo aquel un papel de promotor de las actividades productivas. Esta corriente, como tercer discurso entre la ortodoxia y la heterodoxia tradicional, sostiene la regulación de los movimientos de capital, del tipo de cambio, de la política comercial y de la tasa de interés, con el objetivo de generar un marco macroeconómico estable que sea propicio a la formación de capital y a la adquisición de ventajas competitivas ${ }^{8}$ como medio de aprovechar las oportunidades de inversión e innovación existentes (Bresser Pereira, 2007; Bielchowsky, 2009).

\section{2.b. Dimensión cambiaria y financiera}

La segunda de las dimensiones se vincula a las condiciones de estabilidad macroeconómica y a las modalidades de intervención y manejo de las políticas monetarias y fiscales. La ortodoxia postula una baja intervención sobre el tipo de cambio, el control de la inflación, especialmente a través de tasas de interés altas. Resulta reacia a la emisión monetaria por fuera de las metas macroeconómicas definidas — especialmente la inflacionaria—, a la deuda pública y tiende a la búsqueda del equilibrio macroeconómico (Ortiz Cruz, 2010). El ahorro interno no constituye una preocupación central del modelo ortodoxo, sino que el sistema financiero provee los recursos para el crecimiento de la economía, incluido el financiamiento externo.

El desarrollismo tradicional postula una intervención activa para controlar el tipo de cambio, los precios y la tasa de interés. El Estado opera sobre las

8 Las experiencias neodesarrollistas de la primera década del siglo XXI en América Latina convivieron con elevados precios internacionales de los commodities. Producto de este contexto se observa un aprovechamiento de las ventajas comparativas que acompaña la búsqueda de ventajas competitivas. Esta situación abrió un debate donde se presentan interpretaciones que cuestionan el carácter neodesarrollista y resaltan los rasgos neoextrativistas, asignándole una connotación claramente negativa (GUDYNAS, 2012). Desde nuestro punto de vista, esta 'convivencia' genera una hibridación del modelo que deriva en un buen aprovechamiento de las oportunidades externas, siendo entonces, compatible con el núcleo del proyecto neodesarrollista. 
variables mencionadas para compensar los términos del intercambio, favorecer el consumo de productos nacionales y proteger las industrias locales. Asimismo, asume una posición expansiva de la base monetaria y el control de la tasa de interés para dinamizar el consumo en el mercado interno aunque ejerce cierto control sobre los precios con el objetivo de mantener la inflación contenida dentro de ciertos parámetros y evitar así que esta se dispare.

A diferencia del desarrollismo tradicional, la heterodoxia neodesarrollista no solo le asigna un rol importante a las políticas microeconómicas sino que considera que una política macroeconómica activa es fundamental para alcanzar los objetivos económicos. Sugiere políticas de corte keynesiano a partir de un tipo de cambio competitivo, controles de capitales si fuese necesario, una tasa de interés baja, control de la inflación, una preferencia para conseguir superávits gemelos — comercial y fiscal- y promueve la apertura regulada de la cuenta capital (Bresser Pereira, 2007; Bielchowsky, 2009). De esta forma se busca garantizar un crecimiento económico con ahorro interno sin descartar las oportunidades —atracción de inversiones productivas - que el sistema financiero internacional tiene para contribuir a este objetivo. En esa dirección perciben que la globalización comercial es una oportunidad que tienen los países emergentes para consolidar sus estrategias de desarrollo mientras que la globalización financiera es considerada una gran amenaza para los intereses de este grupo de países.

La hibridación entre la ortodoxia y la heterodoxia tradicional propia del neodesarrollismo se manifiesta, entre otros, en la política fiscal. En este sentido, los neodesarrollistas reconocen la inviabilidad de tener déficit fiscal crónico y, por ende, la necesidad de realizar esfuerzos para alcanzar una estabilidad fiscal. El anclaje de esta política es parte de la propuesta ortodoxa. En lo que respecta al nivel de la tasa de inflación, el neodesarrollismo asume que una inflación moderada es compatible con los objetivos de desarrollo y que resulta necesaria para estimular los niveles de crecimiento económico del país.

\section{2.c. La dimensión social}

Los modelos ortodoxos postulan la reducción del Estado asignándole a este una escasa o nula participación en la resolución de los problemas de tipo social. La distribución del ingreso se rige principalmente por las reglas del mercado. En materia de I+D, infraestructura, etc., se pondera de manera preferencial al sector privado, siendo el Estado un actor subsidiario, especialmente de control de las reglas de juego. 
Los heterodoxos tradicionales consideran que el Estado debe asumir un rol clave en materia de infraestructura y en la provisión de bienes públicos esenciales tales como la salud y la educación, entre los más destacados (Ortiz Cruz, 2010; Bresser Pereira, 2007; Bielchowsky, 2009). El Estado desarrollista debe financiar los bienes públicos a través del ahorro interno forzoso ya que el nivel de ahorro de los agentes privados resulta insuficiente para realizar inversiones de gran envergadura. Esta posición descansa en la convicción de que el ingreso de las IED debe ser limitado en la economía nacional con el objetivo de reducir la capacidad de intervención de los agentes externos.

Los modelos neodesarrollistas estiman que el Estado debe jugar un rol estratégico en materia de inversión, política industrial y social. Apuntan a la creación de infraestructura científica y tecnológica, articulada con los sectores productivos de orientación exportadora, y también a la inversión pública dirigida a la justicia social, buscando amortiguar los impactos de la apertura externa (Banguero, 2004; Bresser Pereira, 2007; Curia, 2011; Bielchowsky, 2009). A diferencia de los ortodoxos, el papel del Estado en el impulso de políticas sociales y transferencia de rentas resulta una variable central para garantizar metas de crecimiento y desarrollo interno. En otros términos, la propuesta neodesarrollista trasciende el enfoque de contención social aplicado por otros modelos de desarrollo al sostener que las políticas sociales forman parte integral de la estrategia de desarrollo que propone. La redistribución del ingreso y la ampliación del mercado interno, a partir de políticas sociales activas y de la concertación entre el capital y el trabajo, es una variable central del modelo (Araníbar y Rodríguez, 2013, p. 54). Dentro del neodesarrollismo las cuestiones energéticas son revalorizadas por su impacto en la sustentabilidad del desarrollo. En el marco de las alianzas público-privadas que caracterizan a esta estrategia, se destaca la complementariedad de las acciones de los agentes no estatales en la provisión de los temas mencionados en esta dimensión.

\section{La vinculación de los modelos de desarrollo según sus tipos ideales con la estrategia de inserción internacional}

La vinculación con la estrategia de inserción cobra diferentes valores según cada uno de estos modelos de desarrollo. Así, el enfoque ortodoxo sostiene que las políticas domésticas relativas al modelo de desarrollo deben ser coherentes y acoplarse con las líneas directrices de la estrategia liberal de inserción internacional teniendo en cuenta las características 
del contexto externo. En la primera dimensión que articula modelo de desarrollo y estrategia de inserción, sobresale el enunciado que sostiene un libre juego de las fuerzas del mercado a nivel interno y en las relaciones comerciales y financieras con el mundo. Luego de la experiencia de las primeras décadas del siglo XX, este discurso vuelve a identificarse en América Latina en los 90 con la implementación de las reformas del Estado a través de los planes de ajuste estructural sugeridos por el Fondo Monetario Internacional (FMI) y el Banco Mundial (BM). Las políticas de apertura y desregulación de la economía suponen la reducción y/o eliminación de las barreras arancelarias y no arancelarias y de los subsidios, con el objetivo de hacer más eficientes los intercambios comerciales y los agentes de producción. En consonancia con ello, promueve la especialización de la estructura productiva basada, principalmente, en las ventajas comparativas.

Asimismo, en el marco de las definiciones del modelo destinadas a alcanzar la estabilidad macroeconómica es necesario construir un escenario doméstico atractivo como destino de inversiones externas y una economía de bajo riesgo que pueda acceder al mercado internacional de capitales para obtener financiamiento de bajo costo.

Teniendo en cuenta las características mencionadas, no es casual que el 'regionalismo abierto' haya sido el modelo de integración preferido por América Latina en los 90 puesto que era compatible con el modelo de desarrollo predominante y con los lineamientos de la estrategia liberal de inserción internacional. La articulación modelo de desarrollo-modelo de integración contribuyó a suavizar los costos de la apertura económica unilateral, reforzó los procesos de liberalización comercial, incrementó los niveles de interdependencia económico-comercial e influyó en la atracción de mayores flujos de inversiones externas hacia los países del bloque MERCOSUR. Desde la ortodoxia se entendió que la participación en estos procesos de integración era la vía para que los países en desarrollo alcanzaran una mejor inserción en la globalización.

Para el pensamiento desarrollista existe una estrecha relación entre el modelo económico y la estrategia de inserción internacional de impugnación del orden internacional. En otras palabras, si no se producen cambios en el sistema internacional, las reformas económicas e institucionales nacionales son insuficientes para los objetivos propuestos por el desarrollismo (Jaguaribe, 1979).

De acuerdo a Bielchowsky (2009), el estructuralismo examina las especificidades productivas, sociales, institucionales y de inserción internacional de los países de América Latina y el Caribe. Se asume el carácter de 'pe- 
riféricos', en contraposición a las características de las economías 'centrales’. La división centro-periferia — la ponderación del clivaje Norte/Sur del orden internacional—, y más específicamente la necesidad de superar la condición periférica, es el núcleo de la estrategia de inserción internacional propuesta por el modelo desarrollista. En este sentido, se cuestiona la visión liberal de los beneficios de la división internacional del trabajo, fundamentando esta crítica en la tesis del deterioro de los términos del intercambio. Dentro de la primera dimensión, dado que la industrialización es una necesidad si se pretende dejar atrás el subdesarrollo, el control del Estado sobre el sector externo es el linkage para avanzar en un modelo ISI. El desarrollismo asume que la liberalización comercial y las políticas de los organismos multilaterales reproducen la lógica Norte-Sur. Por este motivo consideran pertinente crear mecanismos regionales de integración, pero cerrados (stumbling blocks) — como la Asociación Latinoamericana de Libre Comercio (ALALC) y la fallida de Comisión Especial de Coordinación de América Latina (CECLA)_- y de impugnación dentro del sistema multilateral al orden de Bretton Woods. ${ }^{9}$ En cuanto a las definiciones macroeconómicas y el modo de vinculación con el sistema financiero internacional, el carácter cerrado de este modelo de desarrollo le asigna una escasa relevancia a la participación en los organismos multilaterales de crédito, máxime en un contexto de represión financiera. Es decir que, ante un eventual déficit en la balanza de pagos, se adoptaba una política de ajuste endógeno — ciclos stop and go-.

Los neodesarrollistas, por su parte, reconocen una estrecha vinculación con el sistema internacional para cada una de las dimensiones que definen el modelo. Tal vinculación, no obstante, no es inmediata o acrítica sino que resulta mediada por los propios objetivos planteados para alcanzar las metas del desarrollo. Los representantes de este modelo reconocen la puja de poderes que existe dentro del sistema internacional detrás de la definición de cada estrategia de desarrollo. Así, buscan diferenciarse del discurso del Consenso de Washington (Bresser Pereira, 2007) y sostienen, frente a la imposibilidad de alterar los valores del contexto, la necesidad de transformar la relación con el mundo a partir de la propia transformación interna como vía para el desarrollo (Ferrer, 2007, p. 12). Se corresponde entonces con una estrategia reformista de inserción internacional.

9 Un ejemplo de esta impugnación puede encontrarse en la creación y utilización de la UNCTAD como foro alternativo al GATT por parte de los países en desarrollo (SIMONOFF, 2007; TUSSIE, 1993). 
La globalización, dato central del contexto en el cual tanto el pensamiento neodesarrollista como sus experiencias empíricas han tenido lugar (Ferrer, 2013), es considerada como un espacio de oportunidades pero también como una fuente de amenazas. En cuanto a la apertura externa, tanto las exportaciones, como la recepción y emisión de IED son valoradas positivamente. En consecuencia, se dispone una vinculación selectiva y estratégica con el sistema internacional que permita un aprovechamiento de las ventajas competitivas en materia de comercio y producción, para lo cual se recomienda un estilo 'pragmático' en las negociaciones comerciales multilaterales y preferenciales (Banguero Lozano, 2004; Bresser Pereira, 2007). En relación a las definiciones macroeconómicas y financieras, el neodesarrollismo rechaza la apertura total de la cuenta de capitales. Ello se traduce en una política de oposición a los modelos que países desarrollados impulsan dentro de determinados organismos internacionales (Bresser Pereira, 2007) y, al mismo, en la creación de espacios alternativos de cooperación en estas áreas. En la tercera de las dimensiones, la de carácter social, el modelo neodesarrollista destaca en particular las cuestiones de la energía, reconociendo no solo su importancia para la sustentabilidad del desarrollo sino también su carácter geopolítico en el entramado internacional. La integración con Estados semejantes es considerada una vía positiva para mejorar las oportunidades de la globalización, a la vez que para disputar espacios de poder asimétricos (Araníbar y Rodríguez, 2013, p. 21).

\section{Los modelos de desarrollo en la Argentina desde la redemocratización y su articulación con las estrategias de inserción internacional}

Los ejes de vinculación identificados en el apartado anterior se ven reflejados en las distintas experiencias de la PE argentina desde la redemocratización. Tal como se presenta en el siguiente apartado, las continuidades y cambios del accionar externo argentino guardan una correlación con los acoples y desacoples del modelo de desarrollo y la estrategia de inserción internacional.

\section{4.a. El gobierno de Alfonsín}

El gobierno de Raúl Alfonsín estuvo constreñido por la transición en la articulación específica entre el Estado y el mercado, lo que hemos denominado como modelo de desarrollo. En el mundo se producían debates sobre 
el rol del Estado, su naturaleza y forma, como consecuencia de la crisis de los 70, lo que se traducía en una nueva articulación entre el Estado y el mercado bajo el paradigma de la teoría económica neoliberal. En ese contexto, Argentina, tras el fallido experimento neoliberal ensayado por el autodenominado Proceso de Reorganización Nacional (Vázquez Ocampo, 1989), retomaba a comienzos de los 80 los postulados de las políticas heterodoxas.

Una serie de factores se conjugaron para que el gobierno de Alfonsín postulara la necesidad de generar condiciones para la vuelta del Estado como agente de intervención económica. En primer lugar, contribuía a ello la cosmovisión que existía en el Partido Radical sobre la economía. ${ }^{10}$ Asimismo, pesaban los evidentes costos económicos y sociales de la apertura y desregulación de la segunda mitad de los 70. ${ }^{11}$ Por último, condicionaban la decisión los efectos de la crisis de la deuda desatada en 1982.

Sin embargo, como sostiene Liliana de Riz (2001), la administración radical careció de un diagnóstico apropiado del estado de la economía. A la hora de actuar, solo contaba con los instrumentos analíticos y el repertorio de políticas que los radicales habían puesto en práctica con buenos resultados 20 años antes. En ese marco, en una primera etapa del gobierno de Alfonsín existió un retorno a la heterodoxia económica, siendo sus expresiones más acabadas el nombramiento de Grinspun en dicha cartera y las medidas que se comenzaron a aplicar a partir de diciembre de 1983.

Tras los escasos resultados del primer momento 'heterodoxo', el presidente Alfonsín nombró a Juan Vital Sourrouille como nuevo ministro de Economía. El nombramiento del mismo representó un claro giro en materia económica —el denominado "giro realista" — ${ }^{12}$ que se materializó en 1985 a

10 Se debe tener en cuenta que en la ponderación de la política y de la economía, esta estaba en un segundo plano dada la coyuntura de la transición a la democracia y las promesas de encontrar a los responsables de la violación de los derechos humanos en el último gobierno militar. El principal objetivo de la administración Alfonsín era "democratizar a la sociedad argentina”.

11 La fotografía de la herencia económica que dejaba la dictadura militar se traducía en una clara alteración de todos los indicadores: recesión económica, inflación del $600 \%$, una deuda externa de 46.200 millones de dólares equivalentes a casi el $70 \%$ del PBI, desocupación en alza y escasas reservas internacionales (DAMILL y FrENKEL, 1990).

12 Término que utilizó la Cancillería argentina a cargo de Dante Caputo cuando a fines de 1984, en pleno proceso de negociación de la deuda externa, entendió que las diferen- 
través del Plan Austral. Para 1986, el presidente Alfonsín se vio obligado a profundizar los alcances de un plan de estabilización adoptado por necesidad pero muy alejado de sus principios. El gobierno lanzó la segunda etapa del Plan Austral, cuyas medidas apuntaban a impulsar el crecimiento económico, aumentar las exportaciones y privatizar empresas públicas (Escudé y Cisneros, 2000).

A pesar de la voluntad del gobierno de transformar la herencia del proceso militar desde un enfoque desarrollista, las presiones sistémicas y domésticas direccionaron a la Argentina hacia posturas ortodoxas. De acuerdo con Rapoport, el patrón de acumulación que comenzó a perfilarse durante la dictadura se prolongó durante la etapa de Alfonsín, para consolidarse por completo durante la posterior experiencia menemista (Rapoport, 2007).

El principal punto de la estrategia de inserción internacional donde se visualizaron los nuevos condicionamientos liberales fue el tema de la deuda externa, eje central en el vínculo con los EE.UU. (Frohmann, 1986). En un primer momento el gobierno de Alfonsín intentó politizar la temática desde un clivaje Norte-Sur - corresponsabilidad entre acreedores y deudores - apelando a un recurso propio del recetario estructuralista como la creación de mecanismo regionales de integración y/o concertación, tal fue el caso del 'Grupo Cartagena'. La reestructuración de la deuda era la única manera de salir del atolladero sin afectar el nuevo modelo de desarrollo que intentaba impulsar el gobierno radical. Según Frohmann (1986, p. 57) la creencia del gobierno era que "se debían afrontar los compromisos externos sin el agravio al bienestar de los trabajadores ni obstáculos a la reactivación y el desarrollo del país. Esta definición planteaba posturas diametralmente opuestas al ajuste”. Entonces, durante esta primera etapa se identificaba una estrategia de inserción que impugnaba el orden internacional.

Dados los escasos márgenes de poder que la Argentina tenía a la hora de negociar, sumado esto a las necesidades de financiamiento de la economía, se produjo finalmente el 'giro realista' en el vínculo con los EE.UU.

cias con Estados Unidos correspondían a distintas interpretaciones sobre las problemáticas mundiales. Estas interpretaciones no eran un impedimento para la existencia de una relación bilateral madura, en donde Argentina, como país latinoamericano del tercer mundo, reconociera el poderío estadounidense y adscribiera a Occidente, aunque se considerara autónomo en el proceso de toma de decisiones. En este contexto nuestro país intentó un acercamiento hacia Estados Unidos. Muestra de ello fue el viaje que Alfonsín hizo a Norteamérica en el mes de septiembre de ese año. 
Este giro estuvo vinculado a que, fracasada la propuesta de 'regionalizar el tema de la deuda', el apoyo de EE.UU. era clave para las negociaciones del Gobierno argentino con la banca acreedora y para la aprobación de préstamos tanto en el FMI como en el Banco Mundial. A partir del 'acuerdo técnico' que firmó el gobierno de Alfonsín a finales de 1984 con el FMI — para solicitar un préstamo de 1.424 millones de dólares ampliables a otros 200 millones por la previsible caída de exportaciones-, ${ }^{13}$ los desembolsos del Fondo se transformaron en vitales para apaciguar la restricción externa. A contramano de los preceptos desarrollistas, la administración de Alfonsín recurrió al endeudamiento externo para cubrir el déficit de la cuenta corriente.

Asimismo, la imposibilidad del gobierno de Alfonsín de reflotar un modelo de corte desarrollista condicionó la política de integración regional anclada especialmente en la relación con Brasil. De acuerdo con Simonoff (2010), el giro realista de 1985 y el fracaso del Consenso de Cartagena son los dos factores a través de los cuales debe interpretarse el proceso de integración selectiva en el que se enmarca esta relación con Brasil. Tras el importante impulso político que los presidentes argentino y brasileño otorgaron a la cooperación bilateral al firmar la Declaración de Iguazú, en junio de 1986 los gobiernos de la Argentina y Brasil lanzaron el Programa de Integración y Cooperación Económica (PICE). Este tenía como objetivo reducir los desequilibrios comerciales bilaterales con un enfoque sectorial para promover una recuperación de los flujos de comercio a los niveles alcanzados antes de la crisis externa de principios de la década. Como bien destacan Escudé y Cisneros (2000), “para las autoridades del Palacio San Martín, la posibilidad de la integración con Brasil estaba esencialmente vinculada a la necesidad económica de superar el agotamiento del modelo de sustitución de importaciones, impulsando una apertura exportadora estimulada por asociaciones preferenciales”.

En los últimos años del gobierno radical se profundizaron los lazos de integración con Brasil; dicha voluntad se materializó en la firma, el 29 de noviembre de 1988, del Tratado de Integración, Cooperación y Desarrollo, que tuvo lugar durante la visita del presidente Sarney a Buenos Aires. El tratado - piedra angular del futuro MERCOSUR - representó una nueva visión de la integración regional que dejaba atrás los viejos preceptos de ‘regionalismo cerrado’ propio del modelo desarrollista. Según Bouzas y

13 "El Gobierno argentino anuncia la consecución de un «acuerdo técnico» con el FMI”, diario El País, España, 20/9/1984. Disponible en http://elpais.com/diario/1984/ 09/20/economia/464479214_850215.html 
Pagnota (2003, p. 44), "la llegada al poder de gobiernos con un enfoque de política económica más favorable a la apertura a fines de la década del 80, tanto en la Argentina como en Brasil, provocó un cambio sustancial en el contenido de los programas de liberalización; entre estos el principal fue el reemplazo de los mecanismos sectoriales, graduales y flexibles de reducción de aranceles por un procedimiento automático, lineal y generalizado de liberalización”.

En definitiva, durante el gobierno de Alfonsín, dos aspectos centrales de la agenda externa — deuda externa e integración regional— mostraron la complejidad de la articulación existente entre una estrategia de desarrollo en transición y una estrategia de inserción que impugnaba el orden internacional, cuyo principal desafío fue la adaptación a un nuevo orden que se avecinaba. Debido a este permanente ajuste, durante el período en análisis, la estrategia de inserción internacional y el modelo de desarrollo no lograron alcanzar un estado de congruencia y sincronía.

\section{4.b. El Menemismo y la experiencia de la Alianza}

A principio de los años 90, Argentina atravesaba una compleja situación tanto en el plano doméstico como en el plano externo, manifestada en una aguda crisis política y económica, y en una desconexión de sus relaciones con los países desarrollados y con buena parte de los países de la región. En esa coyuntura, fue Carlos Menem quien llevó adelante un proceso de profundos cambios. Esto se evidenció en la implementación de un Plan de Ajuste Estructural en consonancia con el Consenso de Washington y con el aval de los organismos multilaterales de crédito. El mismo se orientó a estabilizar la macroeconomía, frenar la escalada hiperinflacionaria a través del Plan de Convertibilidad y reinsertar al país en el circuito económico y financiero internacional, buscando construir una sinergia particular con los centros económicos internacionales. Es decir que, tras el intento de un modelo híbrido de Alfonsín, el modelo de desarrollo adoptado por el menemismo se ajustó a las características de un modelo ortodoxo.

La estrategia liberal de inserción internacional propuso la redefinición de las relaciones con EE.UU., sustentada en la creencia de que ello redundaría en beneficios económicos relacionados con el aumento del comercio, el restablecimiento del Sistema Generalizado de Preferencias, la atracción de inversiones extranjeras y una mayor fluidez en las negociaciones con la banca privada de capitales y con los organismos multilaterales de crédito. La normalización de los vínculos con Europa supuso la reanuda- 
ción de los intercambios comerciales entre Argentina y la entonces Comunidad Económica Europea, el acceso a líneas de crédito preferenciales y la participación de inversiones europeas en los procesos de privatización de empresas públicas en la Argentina (Rapoport, 2007). Los países limítrofes y América Latina eran visualizados como mercados potenciales para aumentar las exportaciones argentinas con mayor valor agregado, para la atracción de mayores flujos de inversión, para mejorar la competitividad de los productos y conseguir superávit comercial, entre los más destacados. En este sentido, el MERCOSUR fue el instrumento que reflejó tales anhelos.

Durante la primera mitad de los 90, la administración logró una afinada sintonía entre el modelo de desarrollo y la estrategia liberal de inserción internacional en torno a las ideas de la ortodoxia. Ambas variables hicieron eclosión con la crisis mexicana de 1995 y se profundizaron sistemáticamente con las sucesivas crisis en Asia (1997-1998), Rusia (1998) y Brasil (1999), que deterioraron los niveles de crecimiento económico, generaron un aumento progresivo de los niveles de desocupación y, en consecuencia, de la pobreza. Estos sucesos demostraron que la sustentabilidad del modelo ortodoxo basado en el financiamiento del déficit crónico de la cuenta corriente a través de capitales financieros dependía de la liquidez del sistema internacional. ${ }^{14} \mathrm{Al}$ modificarse dicha condición, el modelo alcanzó su límite derivando en un incremento de la vulnerabilidad externa. La recesión de 1997 devino en depresión en los años finales de la década. El aumento de la deuda externa, la ruptura del equilibrio macroeconómico y el aumento de la desconfianza de parte los actores externos sobre el futuro de la Argentina se conjugaron en una combinación explosiva cuyo corolario fue la crisis argentina de 2001-2002.

En las elecciones de octubre de 2000 resultó electo Fernando De la Rúa, candidato por una coalición política, la 'Alianza'. La plataforma electoral postulaba la implementación de ciertas modificaciones a la estrategia liberal de inserción internacional. No obstante, el nuevo gobierno continuó aplicando un modelo de desarrollo ortodoxo. Las políticas de ajuste frente a las sucesivas crisis fueron cada vez más severas y restrictivas en la medida que la economía no mostraba signos de recuperación, el desempleo y la pobreza crecían, las cuentas públicas no encontraban un nuevo punto de

14 Durante los primeros cinco años de la presidencia menemista se produjo el mayor ingreso de IED en el marco de las privatizaciones, lo que contribuía a oxigenar la balanza de pagos. A partir de 1995 el ingreso de tales flujos se contrajo, incrementando el ciclo recesivo. 
equilibrio y el gobierno carecía de fondos por lo que su única opción seguía siendo el endeudamiento externo. Más allá de los lineamientos expresados en la plataforma electoral, el modelo de desarrollo de la Alianza continuó caracterizado por la ortodoxia y la estrategia de inserción internacional liberal mostró solo pequeños giros discursivos y acomodamientos en el modo de gestión antes que en el contenido.

La estrategia de inserción liberal optó por la integración en el Cono Sur pero no abandonó la relación especial con EE.UU. ni con Europa. El ajuste más significativo consistió en pensar la inserción internacional de Argentina desde el MERCOSUR. La continuidad del modelo ortodoxo y de las condiciones externas adversas dificultaron la concreción de cualquier otra alternativa, más allá de las pretensiones discursivas.

En pocos meses el escenario doméstico fue haciéndose cada vez más complejo. Se profundizó la recesión económica y aumentó el endeudamiento externo. Esto fue mostrando un agotamiento cada vez más evidente entre un modelo de desarrollo en crisis y una estrategia de inserción que dejaba de ser funcional a los intereses del país.

La crisis de diciembre de 2001 mostró que la inserción internacional liberal de la Argentina estaba cada vez más condicionada por la incidencia de las cuestiones domésticas y las restricciones externas. Entre las primeras se debe señalar una economía en recesión desde 1997, un altísimo índice de desocupación y un crecimiento exponencial de la pobreza. Los factores externos que afectaban al país se derivaban de la declaración del default ${ }^{15}$ y se expresaron claramente después de 2002 en el proceso de renegociación de la deuda con los organismos multilaterales y los acreedores privados, sumados al cambio de actitud de la administración republicana en EE.UU. respecto de los 'salvatajes' para las crisis financieras, entre otros.

La opción de ambas administraciones por un modelo ortodoxo de desarrollo y su estrategia de inserción internacional liberal solo funcionó aceita-

15 Uno de los corolarios del estallido de diciembre de 2001 fue la declaración de Rodríguez Saa del default de la deuda ante la suspensión del pago del vencimiento de 18 millones de dólares previsto para ese entonces: uno por US\$13 millones con el Fondo Monetario Internacional (FMI) y otro por US\$ 4,3 millones en eurobonos. Para ese entonces la deuda pública de la Argentina ascendía a US\$ 132.143 millones y los títulos públicos (bonos, letras, eurobonos) representaban el 72,2 por ciento del total. La negociación tanto con el FMI como con los tenedores privados de deuda se extendieron durante muchos meses, y que mostraron las tensiones entre el organismo de crédito y la burocracia nacional (Véase NEMIÑA, 2012; LAVAGNA, 2011). 
damente durante la primera mitad de los años 90. Los errores en la gestión de la economía del gobierno de Menem mostraron las primeras fisuras en el funcionamiento armónico de este par de variables. Ellas terminaron de eclosionar durante la administración De la Rúa y se puede inferir que, en esa coyuntura, la decisión de mantener el modelo de desarrollo ortodoxo tuvo un grado de influencia mayor que la estrategia de inserción internacional liberal sobre la política exterior.

\section{4.c. El Kirchnerismo y la experiencia neodesarrollista}

Tras la crisis de 2001, un nuevo cambio se sumó a las recurrentes oscilaciones de la Argentina en cuanto al modelo de desarrollo. La estrategia económica aplicada, primero por el gobierno de Duhalde y luego por los sucesivos gobiernos de Kirchner y Fernández de Kirchner, fue definida en términos de heterodoxa o neodesarrollista. Desde la Presidencia de la Nación, el modelo de desarrollo fue entendido como "la reconstrucción del capitalismo nacional”, basado en "un círculo virtuoso que enlaza la mayor actividad económica, el superávit, la mejor recaudación, la mayor inversión pública, la mejor infraestructura que mejora la competitividad y baja los costos, lo que a su vez retroalimenta el crecimiento económico, en un marco de equidad en el que la educación, la salud y el acceso a una vida digna estén al alcance de todos los argentinos" (Presidencia de la Nación Argentina, 2011).

Agotado el neoliberalismo, el neodesarrollismo de base industrial fue paulatinamente ganando terreno como modelo de desarrollo. Acorde a Porta y Bianco, este debería comprender un fuerte estímulo a la inversión y al consumo, en un mercado interno ampliado hacia el MERCOSUR; el desarrollo de cadenas de valor industriales, sistemas locales de innovación y producción de bienes diferenciados; así como la intervención del Estado a través de políticas activas de promoción, competencia e ingreso y la configuración de redes de aprovisionamiento, en donde se incluya preferentemente a las PyMEs y a la transferencia de la IED (Porta y Bianco, 2004). En este nuevo esquema, a lo largo de la década pudieron coexistir en forma tirante tanto el modelo agroexportador como el modelo industrial, que constituyen tradicionalmente el 'dilema distributivo' ${ }^{16}$ de la economía argentina.

16 Para profundizar sobre los efectos del dilema distributivo en la política comercial véase (ZELICOVICH, 2010). 
Las definiciones centrales del modelo aplicadas inicialmente fueron la búsqueda y mantenimiento de un tipo de cambio competitivo, la promoción de las exportaciones a partir de la adopción de una política comercial externa 'estratégica' ${ }^{17}$ y la obtención de un superávit primario, que implicara crecimiento en base al ahorro interno; también, la reestructuración de deuda externa y la cancelación de los vencimientos con organismos financieros internacionales (Rapoport, 2010), así como la implementación de políticas sociales. ${ }^{18}$ De manera relativamente pronta pudieron verse resultados favorables en cada una de estas áreas. Sin embargo, para el año 2008, tanto por factores externos como internos, el modelo fue encontrando limitaciones y optando por versiones alternativas.

Tras el estallido de la crisis financiera internacional —aunque no solo por ello-, se perdió el carácter competitivo del tipo de cambio y la economía ingresó en un período inflacionario (Curia, 2011). En cuanto a la política comercial externa, esta viró hacia un carácter defensivo, con énfasis en la restricción a las importaciones, el control en la liquidación de divisas y la contracción de las acciones externas en materia de negociaciones internacionales comerciales (Actis y Zelicovich, 2012). La política fiscal no mantuvo, por su parte, los superávits gemelos, lo que incrementó las presiones sobre el comercio exterior. Asimismo, algunos meses antes, el llamado conflicto del campo ${ }^{19}$ había erosionado las bases sociales del modelo. Sobre finales de la primera gestión de Cristina Fernández de Kirchner eran frecuentes los cuestionamientos acerca del real carácter

17 En función de esta definición se diseñaron seis principios rectores para el desarrollo de la PCE: la multipolaridad —entendida como desconcentración geográfica—; la reciprocidad —como el equilibrio entre las concesiones que la Argentina otorga y aquellas que recibe-; el pluritematismo (en términos de diversificación de producto-; la consistencia — vinculada al soporte técnico-; el consenso — modelo de participación abierta-; y la transparencia —distribución de la información- (MECON, 2014).

18 En el caso del modelo de desarrollo aplicado por la Argentina, el gasto público se orientó al frente social, con erogaciones previsionales y aumentos de salarios. Si bien hubo algunas obras de infraestructura impulsadas por el Gobierno, analistas como Rapoport sostienen que no existieron suficientes políticas activas del estado que apuntaran a la creación de sectores de alta tecnología, base de una diversificación de productos y de una plataforma exportadora más avanzada.

19 Enfrentamiento entre los sectores ruralistas y el gobierno de Cristina Fernández de Kirchner suscitado durante el primer semestre de 2008 a partir de la promulgación de una resolución ministerial (RES 125), la cual modificaba la aplicación de los aranceles a las exportaciones de los productos agrícolas. Sobre el conflicto véase LEIRAS y CRUZALEGUI (2009). 
neodesarrollista del modelo de desarrollo ejecutado por dicha administración. ${ }^{20}$

La relación entre el modelo neodesarrollista y una estrategia de inserción internacional reformista fue armoniosa hasta el año 2008, cuando se vio afectada por los cambios señalados. La adopción de un nuevo modelo de desarrollo neodesarrollista se reflejó en un nuevo diseño de la estrategia de inserción internacional, tal como evidencian discursos y acciones del período. En particular, esta vinculación se acentuaba a la hora de buscar diferenciar la gestión asumida en 2003 con la de sus antecesores de corte neoliberal, sin que ello significara romper "un hilo conductor pacifista, latinoamericanista y respetuoso del derecho internacional” (Bielsa, 2005, p. 19) que caracterizaba la vinculación de la Argentina democrática con el mundo. Con las gestiones kirchneristas fueron redefinidas las formas de percibir el contexto internacional, así como los lineamientos que la Argentina debía emprender en el mismo, sus alianzas estratégicas e instrumentos de actuación.

En cuanto al contexto internacional, el gobierno de Néstor Kirchner realizó una impugnación selectiva al orden vigente. Al momento de su asunción se entendió que existían una serie de dificultades tales como "el acceso a los mercados de los países desarrollados, la poca vitalidad de las rondas de negociaciones globales, el peso de la deuda y los legítimos reclamos sociales en un contexto de bajos niveles de financiamiento internacional” (Bielsa 2005, p. 16). Se pensaba que tales constreñimientos podían superarse, en un contexto de globalización, mediante la integración regional. La definición del propio lugar en el mundo fue dada a partir del espacio latinoamericano y como país en desarrollo (Bielsa, 2005). Tal era el lugar que Argentina se definía para sí y privilegiaba del resto de las relaciones (Taiana, 2006, p. 8).

En dicho contexto, las variables económicas adquirieron una importante gravitación para la vinculación de Argentina con el mundo. La inserción internacional fue entendida en términos de comercio exterior e inversiones (Zelicovich, 2011). En sucesivos discursos se sostuvo que la "inserción en el mundo debe ser comercio exterior e inversión extranjera productiva directa” (Kirchner, 2005). Dentro de ello, y en consonancia con las definiciones de la primera de las dimensiones del modelo de desarrollo, el camino a seguir fue definido a través de acciones destinadas a

20 Véase Bresser Pereira (2007), CuRia (2011), Actis y Zelicovich (2012). 
"profundizar la estrategia de apertura de mercados, incrementar sustancialmente nuestro intercambio con el resto del mundo, diversificar exportaciones hacia bienes con mayor valor agregado, desconcentrar ventas por destino y multiplicar el número de exportadores de modo que los beneficios del comercio exterior se derramen sobre todas nuestras ramas productivas” (Kirchner, 2003).

Estas definiciones, junto a la propia percepción de la Argentina como país en desarrollo, contribuyeron a modificar el pensamiento que colocaba a los EE.UU. y a la Unión Europea como sus principales 'socios', especialmente en la esfera comercial y de inversiones, ${ }^{21}$ por el de una orientación más cercana a Brasil y a países como India, Venezuela, Sudáfrica, etc. El MERCOSUR, por su parte, también fue puesto en valor dentro de la estrategia reformista de inserción internacional. Este último adquirió relevancia, tanto comercial como política, al ser "el espacio de ampliación de la autonomía estatal que permite posicionarnos más ventajosamente en el mundo" (Bielsa, 2005, p. 53), incluyendo ello la mejora de "la inserción de nuestro país en el mundo" y la "amplificación de nuestra voz en los distintos foros multilaterales” (Taiana, 2006, p. 6).

La relación con los organismos financieros internacionales fue de confrontación, en tanto que el gobierno no solo mantenía pendiente la resolución de la deuda externa tras la declaración del default, ${ }^{22}$ sino que además impulsaba una visión reformista del régimen internacional. Como alternativa, encontró en los foros multilaterales un espacio para la denuncia de numerosas 'injusticias' del sistema y el tejido de redes de apoyo a las gestiones nacionales para afrontar a los acreedores.

En su conjunto, las definiciones de la estrategia reformista de inserción internacional resultaban coincidentes con el modelo de desarrollo imple-

21 Una vez derrumbado el régimen de convertibilidad (2002), el país — como importante receptor de IED - tuvo un alto grado de conflictividad con las empresas multinacionales (principalmente de EE.UU. y Europa) que habían encabezado el auge de las inversiones en los años noventa. Esa tensión afectó inexorablemente, durante los gobiernos kirchneristas, las relaciones bilaterales con los países de origen de dichas compañías. Algunos casos emblemáticos fueron el de Suez-gobierno de Francia (2005), el de Marsans-gobierno de España (2006) y el de Telecom-gobierno de Italia (2009).

22 Luego de la reestructuración con los bonistas (2005 y 2010) y de la cancelación de la deuda con el FMI, Argentina mantuvo pendiente la resolución de dos frentes en materia de deuda: el del club de París y el de los bonistas que no ingresaron al canje (holdouts). Ambos constituyeron un fuerte condicionante del modelo de desarrollo y de la estrategia de inserción en el período estudiado. 
mentado. Esta coincidencia, incluso, llegó a conducir a analistas como Miranda (2012) a sostener una interpretación de la política externa como sobredeterminada por las variables domésticas. ${ }^{23}$ La crisis financiera internacional supuso varias rupturas, ya señaladas, sobre el modelo de desarrollo que generaron ciertas disonancias en la estrategia de inserción internacional.

Dentro de la dimensión productiva, en el área comercial, al adoptarse un enfoque proteccionista del comercio, se produjeron los ajustes más importantes. Este nuevo enfoque llevó a una menor ponderación del vínculo con MERCOSUR y a una retracción en los ámbitos de negociación multilaterales y preferenciales en donde se exploraba la búsqueda de oportunidades de negocios para sectores estratégicos. En términos de las orientaciones y lineamientos, a medida que se desdibujaba el modelo neodesarrollista, el MERCOSUR dejó de ser "la vía privilegiada para concebir la inserción internacional, surgiendo en cambio otro tipo de relacionamientos bilaterales y la apelación a la cooperación internacional en lugar de la integración regional como respuesta a los problemas colectivos” (Zelicovich, 2011, p. 188). También se vieron afectadas las bases domésticas para el accionar externo, a partir del conflicto puntual de las restricciones a las exportaciones y los aranceles a las exportaciones agrícolas, lo que produjo la primera de las rupturas de la base de apoyo del kirchnerismo (Simonoff, 2010).

La vinculación con los organismos financieros internacionales mantuvo más bien rasgos de continuidad y, tras 2008, se adoptó una posición crítica y reformista de los mismos — coincidente con el enfoque neodesarrollista-, encontrándose un nuevo ámbito de actuación a partir de la reactivación del G20. En el mismo, la presidenta sostuvo “posiciones claramente neokeynesianas" que "tuvieron como destinatarios tanto al frente interno como externo” (Simonoff, 2013, p. 164).

En la tercera de las dimensiones pudo apreciarse un ajuste en cuanto al reposicionamiento de los temas energéticos dentro de la agenda de inserción internacional de Cristina Fernández de Kirchner, aunque este sería más evidente en su segundo mandato (2011-2015). ${ }^{24}$

23 Sostiene Miranda (2012, p. 90) “La política exterior de Kirchner se caracterizó por plantear a esta política desde una modalidad etnocéntrica. Contrariamente a lo que a menudo se sostiene, la política exterior, más que estar dirigida hacia la política doméstica, se había convertido en una proyección de esta a nivel internacional. Prácticamente fue un ejercicio de internacionalismo liberal, desde el cual — como es sabido— se postula que la política interna decide el curso de las relaciones exteriores del país”.

24 Véase LORENZINI (2011). 
En su conjunto, en la política exterior primaron las situaciones de sincronía entre el modelo de desarrollo neodesarrollista y la estrategia reformista de inserción externa. Cuando estas perdieron congruencia, fue el modelo de desarrollo el que mantuvo su primacía, obligando a los ajustes en la estrategia de inserción internacional y afectando el diseño y ejecución de la política exterior.

\section{Reflexiones finales}

El análisis conjunto de la estrategia de desarrollo y modelo de inserción resulta ser una herramienta útil para la comprensión de la PE de países como la Argentina. Conforme lo expuesto a lo largo de este trabajo, es a través de ese par conceptual que resulta posible entender y explicar las continuidades y, especialmente, los cambios en dicha política pública.

El análisis de las administraciones de Alfonsín, Menem-De La Rúa y Kirchner-Fernández de Kirchner muestra que durante las últimas tres décadas se sucedieron reiterados cambios en la dupla conceptual analizada en este trabajo. Los pasos del estructuralismo al neoliberalismo, primero, y luego de este al neodesarrollismo generaron vaivenes en las estrategias de inserción internacional. La relación con EE.UU., las definiciones referidas al endeudamiento externo o la manera de concebir la integración regional ilustran esas modificaciones. Los tres períodos, por su parte atravesaron instancias de crisis. De manera coincidente, en cada una de estas fue la variable del modelo de desarrollo la que terminó primando por sobre la estrategia de inserción internacional, impactando con mayor fuerza sobre la política exterior.

Por tal motivo, un análisis conjunto de la estrategia de desarrollo y de inserción internacional resulta ser una herramienta útil para la comprensión del accionar externo de la Argentina, máxime si el estudio se realiza transversalmente en un período considerable de tiempo. En ese sentido, el principal aporte del trabajo radica en comprender y explicar las continuidades y especialmente los cambios de cómo la Argentina ha transitado su vinculación con el mundo.

A lo largo del trabajo se plantearon tres tipos ideales de estrategias de desarrollo - o sea, de articulaciones específica del Estado y el mercado- las cuales han tenido un correlato, aunque no de manera pura, con las tres etapas descriptas. Una de las particularidades del caso argentino en comparación con otras experiencias latinoamericanas radica en los pocos consensos internos sobre las dimensiones básicas en torno al desarrollo. La Argentina sigue transitando por un "empate hegemónico" en el sentido de que ningún 
proyecto de desarrollo ha logrado imponerse como "ganador". Esta situación provoca oscilaciones y políticas pendulares a la largo del tiempo, agravadas por las vulnerabilidades propias que tiene la globalización para los países periféricos.

Como quedó de manifiesto a lo largo del trabajo, la estrategia de inserción internacional de cualquier país cobra diferentes particularidades según cada uno de estos modelos de desarrollo. Si bien los lineamientos y las orientaciones generales que adquiere el accionar externo de un país no se reduce a este condicionante específico, no es menor el dato de que los impactos en el caso argentino son significativos. De ahí la necesidad de estudiar la relación entre estas dos variables para comprender por qué nuestro accionar externo es tan disruptivo en el tiempo.

En el período 1983-2011 los cambios más acentuados en la PE han estado vinculados a la relación con la potencia hegemónica -los EE.UU.la ponderación de los lazos con los socios regionales y la valoración de los organismos económicos internacionales —encabezado por el FMI-. Cada uno de las etapas en las que dividimos el estudio muestra cambios en estas tres áreas de cuestiones. Encontramos que cada uno de esos cambios puede explicarse por la articulación entre modelo de desarrollo y estrategia de inserción internacional. Como vimos, en el gobierno de Alfonsín se produce un "giro realista" sobre la PE. Dicho giro, que comprende el cambio en los vínculos con los EE.UU. y con el FMI, solo puede entenderse en el marco del agotamiento del modelo desarrollista y la paulatina adopción de un modelo crecientemente neoliberal a partir del plan Austral. En el gobierno de Menem se observa, además, un cambio en el lugar dado a los socios regionales y fundamentalmente en la constitución del MERCOSUR. Es desde el neoliberalismo desde donde se entiende la incorporación de los acuerdos comerciales regionales y asimétricos como mecanismos de inserción en una globalización que se percibe como favorable al crecimiento argentino. En el caso de los gobiernos de Kirchner y de Cristina Fernández de Kirchner es, en cambio, a partir de la consolidación del modelo neodesarrollista que la PE vira hacia una crítica moderada de la globalización, el alejamiento del FMI y el enfriamiento de las relaciones con EE.UU., los que se combinan con una redefinición de los vínculos regionales, en el marco de la priorización del eje Sur-Sur.

La falta de consensos en torno al modelo de desarrollo es la principal amenaza a la continuidad de las políticas públicas en Argentina, y dentro de estas, de la política exterior. Sin acuerdos acerca de la relación Estado-mercado, periódicamente sufrirá de oscilaciones, refundaciones y reformas el accionar externo de la Nación. \$ \$ 


\section{Bibliografía}

Actis, E. y Zelicovich, J. (2012). “Crisis de los modelos neodesarrollistas del MERCOSUR: Argentina y Brasil” ponencia presentada en X Congreso Nacional y III Congreso Internacional sobre Democracia, organizado por la Facultad de Ciencia Política y Relaciones Internacionales de la Universidad Nacional de Rosario.

Actis, E.; LoRenZini, M. E.; Zelicovich, J. (2014). "Modelo de Desarrollo y Estrategia de Inserción: Claves para la interpretación de su relación”, Trabajo presentando en el XI Congreso Nacional y IV Congreso Internacional sobre Democracia, Facultad de Ciencia Política y Relaciones Internacionales-UNR, Rosario, 10 de septiembre

Araníbar, A. y Rodríguez, B. (2013). Latinoamérica, ¿del neoliberalismo al neodesarrollismo. Buenos Aires: Siglo XXI.

BAnguero LozAno, H. (2004). "Nuevas corrientes en la teoría y la política económica entre el neoliberalismo y el neoestructuralismo”. En Revista de Economía y Administración, 1, (1), 93-110.

BÁrcena, A. y Prado, A. (2015). Neoestructuralismo y corrientes heterodoxas en América Latina y el Caribe a inicios del Siglo XXI. Santiago de Chile: CEPAL-IDRCCRDI.

Benort, K. (1995/6). “Democracies really are more pacific (in general); Reexaming Regime Type and War Involvement”. Journal of Conflict Resolution, 40, (4), 636-657.

BielchowsKy, R. (2009). "Sesenta años de la CEPAL: estructuralismo y neo-estructuralismo”. Revista de la CEPAL, 97, 173-194.

Bielsa, R. (2005). “La política exterior argentina”. En BIELSA, R.; RosatTi, H. y LAVAGNA, R. (eds.) Estado y Globalización. El caso argentino. Buenos Aires: Rubinzal Culzoni.

Bresser Pereira, L. (2007). “Estado y mercado en el nuevo desarrollismo”. En Nueva Sociedad, 210, 110-125.

CEPAL (1998). “Cincuenta años. Reflexiones sobre América Latina”. En Revista de la CEPAL, Número Extraordinario, 9-297.

Cervo, A. (2003). "Political Regimes and Brazil’s Foreign Policy”. En Sombra SaraiVA, J. F. (Ed.). Foreign Policy and Political Regimes. Brasilia: IBRI, 341-362.

CuRIA, E. (2011). El modelo de desarrollo en Argentina. Los riesgos de una dinámica pendular. Buenos Aires: Fondo de Cultura Económica.

Devlin, R. y Moguillansky, G. (2009). “Alianzas público-privadas como estrategias nacionales de desarrollo a largo plazo”. En Revista de la CEPAL, 97, 97-116.

Di FILIPPO, A. (2007). "La Escuela Latinoamericana del Desarrollo: Tensiones Epistemológicas de un movimiento fundacional”. En Cinta de Moebius, 029, pp. 124-154.

Di Pace, M. (Coord.) (1992). Las Utopías del Medio Ambiente. Desarrollo Sustentable en la Argentina, Buenos Aires: Centro Editor de América Latina.

Doyle, M. (1983). “Kant, Liberal Legacies, and Foreign Affairs”. En Philosophy and Public Affairs, 12, (3), 205-235.

(1986). "Liberalism and World Politics". En The American Political Science Review, 80 (4), 1151-1169. 
Escudé, C. y Cisneros, A. (2000). "Historia de las Relaciones Exteriores Argentinas”. (Consulta 14/03/2015) (on line). Disponible en http://www.argentina-rree.com/ historia_autores.htm

Fernández Bugna, C. \& Porta, F. (2008). "Crecimiento reciente de la industria argentina. Nuevo régimen sin cambio estructural”. En Kosacoff (Ed.). Crisis, recuperación y nuevos dilemas. La economía argentina 2002-2007. Documento de Proyecto $\mathrm{N}^{\circ}$ 20, CEPAL, 17-48.

Ferrer, A. (2007). "Prólogo”. En Girón y CorreA, Del Sur Hacia el Norte. Economía Política del Orden Económico Internacional emergente. Buenos Aires: CLACSO.

_. (2005). “Globalización, desarrollo y densidad nacional”, Documento de la Fundación Políticas Públicas (on line), (Consulta 16/06/2014). Disponible en http:// www.politicaspublicas.org.ar/pdf/fpp_ferrer_28.04.pdf

Ferrer, A. (2011). "Prólogo”. En CuriA, E. El modelo de desarrollo en Argentina. Los riesgos de una dinámica pendular. Buenos Aires: Fondo de Cultura Económica.

. (2013). "La importancia de las ideas propias sobre el desarrollo y la globalización”. En Revista Problemas del Desarrollo, 173, (44), 163-173.

FFrench DAVIs, R. (2001). Entre el neoliberalismo y el crecimiento con equidad. Tres décadas de política económica en Chile, Dolmen-CIEPLAN, Santiago de Chile.

Frenkel, R. (2003). “Globalización y crisis financieras en América Latina”. En Revista de Economía Política, 23, (3), 94-111.

Frohmann, A. (1986). "Democracia, deuda externa y disciplinamiento económico. Las relaciones entre Argentina y EE.UU. 1983-1985”, Documento de Trabajo de FLACSO, $\mathrm{N}^{\mathrm{o}} 298$.

Gaubatz, K. T. (1996). "Democratic states and commitment in international relations”. En International Organization, 50, (1), 109-139.

GudynAs, E. (2012). "Estado compensador y nuevos extractivismos. Las ambivalencias del progresismo sudamericano”. En Nueva Sociedad, 237.

Hurrell, A. (1995). “O ressurgimento do Regionalismo na Politica Mundial”. En Revista Contexto Internacional, 17, (1), 23-59.

(2003). "Political Regimes and Foreign Policies: An Introduction”. En SomBra SARAIVA, J. F. (Ed.). (2003). Foreign Policy and Political Regimes. Brasilia: IBRI, 29-64

JAGuARIBE, H. (1969). “Dependencia y autonomía en América Latina”. En VARIos AutoRES, La dependencia político-económica de América Latina. México: Siglo XXI.

Kaldor, N. y MirrleEs, J. A. (1962). “A new model of economic growth”. En The Review of Economic Studies, 29, 174-192.

KIRCHNER, N. (2003). Discurso ante Asamblea Legislativa en ocasión de la toma de posesión del cargo de presidente de la Nación Argentina. 25 de mayo de 2003.

Kirchner, N.y Di Tella, T. (2003). Después del derrumbe. Buenos Aires: Galerna.

LaVAgnA, R. (2011). El desafío de la voluntad. Trece meses cruciales en la historia de argentina abril 2002-mayo 2003. Buenos Aires: Editorial Sudamericana.

LeIRAs, M. y CRUZALEgUI, I. (2009). “Argentina: problemas macro-económicos, conflicto social y debilitamiento de la coalición de gobierno”. En Revista de Ciencia Política, 2, (29). 
Lorenzini, M. E. (2011). Política Exterior, Alianzas Estratégicas y Energía en América Latina. Las relaciones argentino-chilenas bajo la lupa. Rosario: Homo Sapiens.

. (2012). "La inserción internacional de la Argentina pos crisis: Impactos sobre sus vínculos con Chile”, Trabajo presentado en el Seminario Argentina/Chile-Chile/ Argentina. Compartiendo y comparando agendas, Consulado de Chile en RosarioFacultad de Ciencia Política y Relaciones Internacionales, 28 de agosto.

Míguez, M. C. (2013). Los partidos políticos y la política exterior argentina. Buenos Aires: Ariel.

MirandA, R. (2012). “Des-inserción argentina. Las políticas exteriores de Menem y Kirchner”. En Revista Enfoques: Ciencia Política y Administración Pública, X, (17), 85-103.

MoravcsiK, A. (2002). "Liberal International Relations Theory: A Scientific Assesment”. En Elman, C. \& Fendius Elman, M. (Ed.). Progress in International Relations Theory. Cambridge: MIT Press.

NemiñA, P. (2012). “Notas para el análisis estratégico de los procesos de negociación entre el FMI y los Gobiernos tomadores de créditos”, ponencia presentada en la VII Jornadas de Sociología - UNLP, La Plata, Diciembre 2012.

OMAn, C. (2010). Policy Competition for Foreign Direct Investment: a Study of competition among Governments to Attract FDI. París: Organización de Cooperación y Desarrollo Económicos.

Ortiz Cruz, E. (2010). “Modelos de desarrollos heterodoxos y ortodoxos”. En Revista Economía UNAM, 7, (19), 69-790.

RAPOPORT, M. (2010). Las políticas económicas de la Argentina. Una breve historia. Buenos Aires: Booket.

. (2006). Historia económica, política y social de la Argentina 1880-2003. Buenos Aires: EMECÉ.

RAPOPORT, M. y MíGUEz, M. C. (2015). “Desafíos y ejes para una inserción internacional autónoma de la Argentina y América del Sur en el escenario internacional”. En BRICEÑo Ruiz, J. y Simonoff, A. (Comp.) Integración y Cooperación Regional en América Latina. Biblos. Buenos Aires, 143-162.

RApoport, M. \& Siguel, C. (2003). "Modelos Económicos, Regímenes Políticos y Política Exterior Argentina”. En Sombra Saraiva, J. F. (comp.), Foreign Policy and Political Regime, Brasília: Instituto Brasileiro de Relações Internacionais (IBRI), 169-236.

Simonoff, A. (2007). "Los dilemas de la autonomía: La política exterior de Arturo Illia (1963-1966)”. Tesis doctoral presentada para la obtención del título de Doctor en Relaciones Internacionales, Facultad de Ciencias Jurídicas y Sociales, Universidad Nacional de La Plata.

(2010). La Argentina y el mundo frente al bicentenario de la Revolución de Mayo: las relaciones exteriores argentinas desde la secesión de España hasta la actualidad. La Plata: Universidad Nacional de La Plata.

. (2012). "Seis claves explicativas para la política exterior argentina durante el primer mandato de Cristina Fernández (2007-2011)”. "Ponencia presentada en VI Congreso de Relaciones Internacionales del IRI, La Plata. 
. (2013). “Análisis estructural de la estrategia de inserción internacional del primer gobierno de Cristina Fernández (2007-2011)”. En Revista Relaciones Internacionales, 44.

Sombra Saraiva, J. F. (Ed.) (2003). Foreign Policy and Political Regimes. Brasilia: IBRI.

STIGLITZ, J. (1998). “Towards a new paradigm for development: strategies, policies and processes”, Presentación "Prebisch Lecture”. Ginebra: UNCTAD, 19 de octubre.

TAiAna, J. (2006). “Objetivos y desafíos de la política exterior argentina”. En Diplomacia, Estrategia, Política, 4, 5-16.

Tussie, D. (1993), Los países menos desarrollados y el sistema de comercio mundial, México, D.F.: Fondo de Cultura Económica.

VÁzQuez OCAMpo, J. M. (1989). Política Exterior Argentina: de los intentos autonómicos a la dependencia (1973-1983). Buenos Aires: Centro Editor de América Latina.

ZeLicovich, J. (2010). "El dilema distributivo y la política exterior argentina en la Ronda Doha”, Ponencia presentada en el V Congreso de la Asociación Latinoamericana de Ciencia Política (ALACIP), Buenos Aires.

. (2011). "El lugar del MECOSUR en la Política Exterior Argentina durante los gobiernos de Néstor Kirchner y Cristina Fernández de Kirchner”. En Revista Relaciones Internacionales, 20, (41), 179-195.

Fecha de recepción: 14/10/2016

Fecha de aceptación: 19/02/2017 